\title{
Fault classification in smart distribution network using support vector machine
}

\author{
Ong Wei Chuan ${ }^{1}$, Nur Fadilah Ab Aziz ${ }^{2}$, Zuhaila Mat $\operatorname{~asin}^{3}$, Nur Ashida Salim ${ }^{4}$, \\ Norfishah A. Wahab ${ }^{5}$ \\ ${ }^{1,2}$ Department of Electrical and Electronics Engineering, Universiti Tenaga Nasional, Malaysia \\ ${ }^{3,4,5}$ Faculty of Electrical Engineering, Universiti Teknologi MARA, Malaysia
}

\begin{tabular}{l}
\hline Article Info \\
\hline Article history: \\
Received Oct 2, 2019 \\
Revised Dec 4, 2019 \\
Accepted Dec 18, 2019 \\
\hline
\end{tabular}

Keywords:

Distribution network

Fault identification

Smart meter

Support vector machine

\begin{abstract}
Machine learning application have been widely used in various sector as part of reducing work load and creating an automated decision making tool. This has gain the interest of power industries and utilities to apply machine learning as part of the operation. Fault identification and classification based machine learning application in power industries have gain significant accreditation due to its great capability and performance. In this paper, a machine-learning algorithm known as Support Vector Machine (SVM) for fault type classification in distribution system has been developed. Eleven different types of faults are generated with respect to actual network. A wide range of simulation condition in terms of different fault impedance value as well as fault types are considered in training and testing data. Right setting parameters are important to learning results and generalization ability of SVM. Gaussian radial basis function (RBF) kernel function has been used for training of SVM to accomplish the most optimized classifier. Initial finding from simulation result indicates that the proposed method is quick in learning and shows good accuracy values on faults type classification in distribution system. The developed algorithm is tested on IEEE 34 bus and IEEE 123 bus test distribution system.
\end{abstract}

Copyright $@ 2020$ Institute of Advanced Engineering and Science. All rights reserved.

\section{Corresponding Author:}

Nur Fadilah Ab Aziz,

Department of Electrical and Electronics Engineering,

Universiti Tenaga Nasional, Selangor, Malaysia.

Email:nfadilah@uniten.edu.my

\section{INTRODUCTION}

Power distribution system (PDS) is a power system which delivers power from the transmission system to consumers or residential customers. PDS contains a complicated structure and difference in voltage levels. PDS is separated into two parts which are primary and secondary distribution system. The primary distribution system has medium voltage levels of $11 \mathrm{kV}$ and $33 \mathrm{kV}$ which are directly distributed to heavy loads and uses in of industries. Secondary distribution system uses low voltage level with 400V and 230V where the domestic consumer voltage usage [1].

PDS frequently works in radial structure with a combination of distribution transformer (DT), bus lines, switches, fuses and unbalanced loads. PDS is connected to house loads or regular loads from the primary substation using DT to step down to a low voltage level. Industrial loads which are above $11 \mathrm{kV}$ are directly supplied by the primary grid which is an interconnected network that delivers power from the substation to DT. These loads have a greater voltage level. Furthermore, secondary grid distributes power from DT to regular loads by various routes to avoid fault occurrences. Any disturbances in PDS will cause an outage in the distribution system. Most of the outage is caused by the different types of faults that exist on the line for an extended period. Any components failure in PDS will cause outage directly. 
Faults in a distribution system can be either single phase to ground, double phase to ground, phase to phase, three phase to ground or three-phase faults [2]. Each of these faults can be distinguished by their characteristic e.g. Single phase to the ground fault will give a spike in the current values of the particular phase during the fault occurrence. Some of the fault location methods will require the identification of fault type in prior before estimation of the fault location. Another aspect which can be related to fault type is the fault level. Fault level can be divided into two different kinds which are low impedance and high impedance fault. Low and high impedance fault are distinguished by the fault current values resulted from the arching between the phase to the surface of contact. Unlike low impedance, high impedance faults are difficult to be detected [3]. It requires customized approaches that can extract information of these high impedance faults from the available data.

The reliability, security and power quality of distribution system are significantly affected by distribution fault. Faults occurrence in distribution system is difficult to avoid and unpredictable during steady state conditions. Although, the disturbance can be found all over of the power system, but almost $80 \%$ of fault related interruption experienced by customers are due to fault incidents on the distribution system [4]. The occurrence distribution outage due to the fault will affect the utilities' reliability index. So, in order to full fill the reliability index criterion, utilities have to devise fault identification method which ensures power is restored to users in short duration of time. Identification of fault types is very crucial for the protection scheme to maintain distribution system stability and minimize customer minute losses and network damages. Hence, quick faults type recognition within minimum error allows the relays and circuit breakers to operate and isolate the faulty part to protect the components and therefore the efficiency of the power distribution system can be increased. Consequently, fast and reliable fault detection has become an important operational demand in distribution system.

The classical method of fault identification process in power system employed by utilities is a step by step process. First, the affected area of disturbance is identified through customer phone calls. This enables utilities to generally localize the disturbance location and its affected customer. After having sufficient geographical and statistical data, the maintenance teams are dispatched to affected substation. They will conduct visual inspections together with measurement to estimate the cause of the disturbance. After identifying the cause of the disturbance, if it is due to fault occurrence, methods such as capacitive discharge and pulse echo meter will be used to obtain the estimated location of fault [5].

Basically, the classical method of fault identification requires more time for restoring the power. Besides, the classical method also contributes to higher operation and maintenance cost for the utilities. In order to overcome this problem, modern methods of fault identification have been researched and developed. Quick faults type recognition within minimum error allows the relays and circuit breakers to operate and isolate the faulty part to protect the components and therefore the efficiency of the power distribution system can be increased. Consequently, fast and reliable fault detection has become an important operational demand of MV distribution system.

Smart meter data provides a better understanding of how distribution networks operate and assist in the more active management of the networks by enabling two-way communication between the meter and the central distribution system. Smart meters can measure the voltages and loads through wireless communication. When any fault occurs in the distribution network, sequence currents and voltages are generated and can be captured by the smart meter. In this paper, a fault classification algorithm using a machine learning known as Support Vector Machine (SVM) has been developed. The data of current and voltages employed can be collected by a smart meter. The data is used as the training, testing and validation data of the SVM.

\section{MODERN FAULT CLASSIFICATION TECHNIQUES}

\subsection{Smart Meter}

The main problem of fault events in the distribution system is that the insufficient information that exists on the metering point. Recently, smart meters are developing and can be used to overcome the fault analysis in the distribution system. A smart meter is an energy meter that used to measure electrical data and record reading with more accurate than current conventional energy meter. The two-way communication used in a smart metering system which known as Advanced Metering Infrastructure (AMI) able to monitor the meters from the control room [6]. Smart meters can provide data of energy consumption of customer and notify the utility grid when there is a fault occurs [7].

There is a proposed method that needed voltage sag data which the data is collected from the smart meter that installed along the feeder at a strategic point. This method used to capture voltage drop during fault condition which has different in magnitude than the pre-fault condition. On-fault measurement is used to verify the voltage of each node with the help of reverse and forward sweep load flow to measure the fault current for each node in an iterative loop. The fault current is to determine the faulted node with the least 
error between actual measurement and calculated voltage drops. Unknown voltages from previous shortcircuit analysis calculations are introduced and errors in the measurements that resulted in not properly estimate the faulted node. This method showed that it was crucial of collecting pre-fault and on-fault data from smart meters in order to identify software-based fault types. The data obtained by the smart meters can be collected in order to have real-time monitoring of identification of fault location [8].

The fault type method based on state estimation is proposed in [9] which require on-fault currents of the supply and on- fault voltage profiles of buses that can be obtained from smart meters and transferred to the control centre to justify the type of fault. The data collected by the smart meters can be processed in order to have monitoring of fault types classification in real-time. This method achieves better results with just one radial feeder in a single-line distribution system.

\subsection{SVM as the Application of Machine Learning}

Recently, machine learning has been speedily developed and applied successfully to classify types of fault on either transmission or distribution system [10]. However, majority of the research focus on the fault identification for overhead power transmission system [9-15] but not for the power distribution system. A quick method to predict the fault occurrence is needed since an instant operation is required to avoid the power distribution system from an outage. SVM is relatively a classification method that shown higher performance and accuracy than other learning methods in a lot of application [16].

A. Ngaopitakkul and C. Pothisarna proposed a method to identify the types of faults in underground PDS using a combination of discrete wavelet transform (DWT) and SVM [17]. Fault detection on highfrequency component from the fault signals by using the DWT algorithm. Positive sequence current of fault signals is used as input for the SVM in a decision algorithm. Several case studies with the difference of fault types and fault impedance have been worked out. Some researchers have employed smart meter data in support vector machine (SVM) for fault location in power system. In reference [18], an artificial neural network (ANN) and SVM was proposed for locating faults in radial distribution systems. In reference [19], SVM is used to predict fault location in PDS with distributed generation (DG) and smart meter. The study uses a fixed value of SVM parameter with the chosen RBF as the Kernel function and cross validation method. In the RBF kernel, there are two parameters which are $\mathrm{C}$ and gamma $(\gamma)$ needs to be selected as the best parameters in order for SVM to predict the test data accurately. One method proposed by Livani used SVM based learning algorithm to identify the fault location on faulted lines [20]. The proposed method used RMS voltage data from smart meter as the input for SVM. Several authors also proposed methods which used machine learning algorithm together with data from other equipment besides the smart meter. A study performed by Agrawal used both SVM classification and regression for fault type and location [21]. The input data for fault classification were obtained from a power quality recorder. Meanwhile, Deng used current waveform as the input data for SVM [22]. The developed method was used to identify the exact location of fault in a power line. From literature review, it is observed that majority of the proposed algorithms for fault classification and location were tested on small bus test distribution system only. This paper aims to test the SVM for fault classification in both small and big test distribution system.

\section{FAULT CLASSIFICATION USING SVM}

In order to detect the fault types in a short period for faster fault isolation and increased speed of distribution power system restoration process, a machine learning technique using SVM with the implementation of the smart metering is introduced. Simulation-based analysis using a software program known as OpenDSS has been adopted to run fault analysis for data generation under different fault types of a distribution system.

\subsection{Fault Simulations}

The network faults were simulated in the OpenDSS software [23]. The initial progress started off with the initialization of all the variables in the software. Simulations of pre fault and on-fault conditions were conducted and the output data from pre fault was used to validate the data of on-fault conditions. 11 types of fault condition comprise of single line to ground, double line to ground, line to line and three phase faults with 9 different fault impedances were simulated as the input data for SVM. Voltage data was used as the machine learning input. Figure 1 shows the flowchart of data generation through fault simulations using OpenDSS software.

\subsection{Support Vector Machine (SVM)}

SVM is an intelligent tool and known as a new machine learning technique which is a statistical learning method with a powerful theoretical foundation based on Vapnik-Chervonenkis theory. 
SVM has been successfully applied to classification, regression, and density estimation problems [16]. The fundamental theory of Support Vector Machine is described as shown in (1) and (2) [24]:

$$
\begin{aligned}
& \left\{\left(x_{1}, y_{1}\right),\left(x_{2}, y_{2}\right),\left(x_{3}, y_{3}\right) \ldots,\left(x_{L}, y_{L}\right)\right\} \text { with } x_{i} \in R_{n} \\
& \mathrm{y}_{\mathrm{i}} \in\{-1,+1\}
\end{aligned}
$$

Where

$\mathrm{x}=$ system input

$\mathrm{y}_{\mathrm{i}}=$ the system output

As eloquently stated by Vapnik's formulation, SVM classifier can be formulated as (3):

$$
f(x)=\mathrm{w}^{T} \varphi(x)+\mathrm{b}
$$

Where

$\psi(\mathrm{x})=$ non-linear function that maps the input $\mathrm{x}$ into feature space

$\mathrm{w}^{T}=$ m-dimensional vector

$\mathrm{b} \quad=$ the system output

In order to divide the data into the feature space linearly, the decision function must satisfy certain conditions which as shown in (4):

$$
\mathrm{y}_{\mathrm{i}}\left(\mathrm{wT} \varphi\left(\mathrm{x}_{\mathrm{i}}\right)+\mathrm{b}\right) \geq 1
$$

Where $i=1, \ldots \ldots ., L$

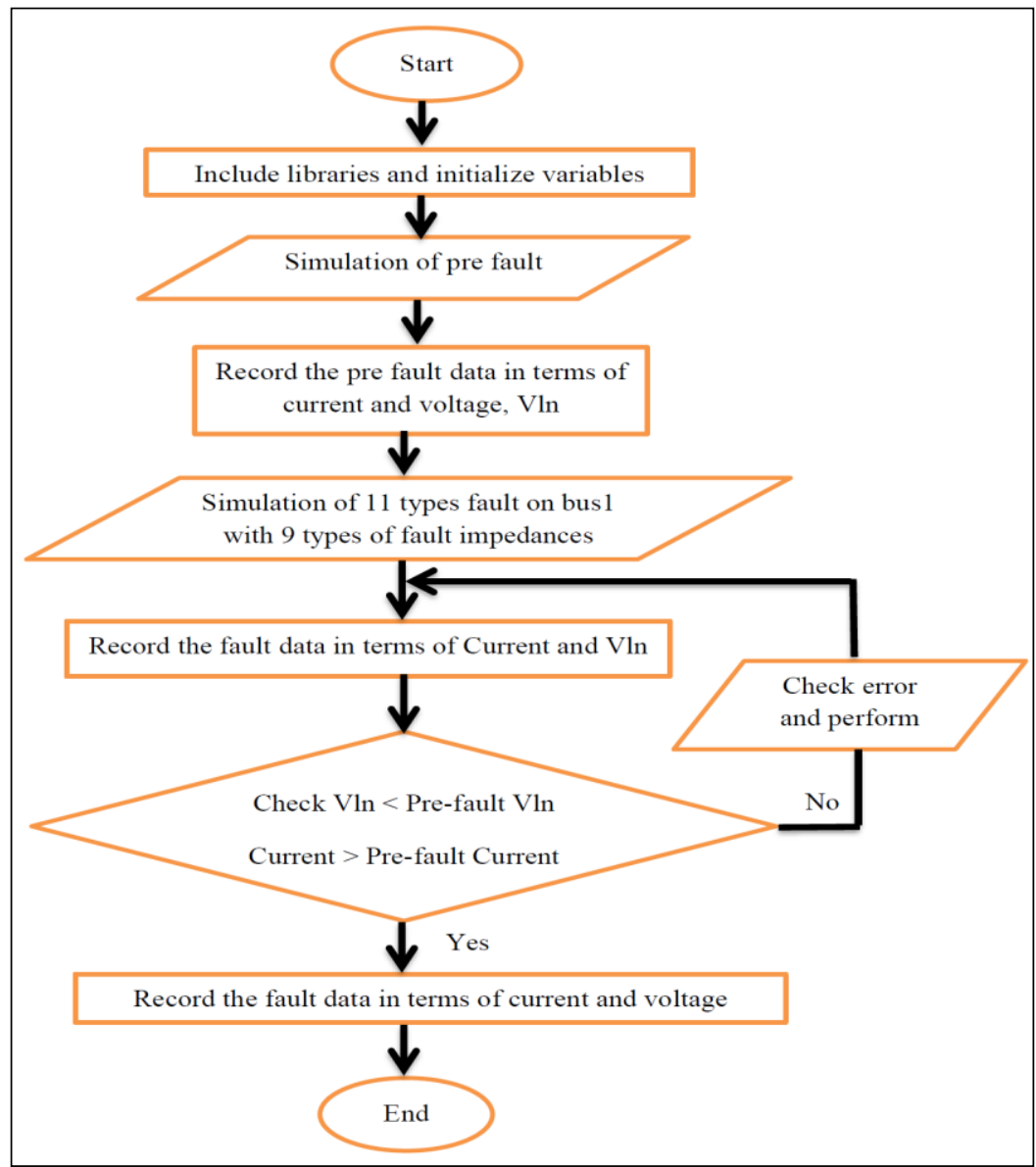

Figure 1. Flowchart of Fault Simulations in OpenDSS 


\subsection{SVM Parameter Selection}

In order to develop a SVM model, several parameters must be specified:

a) The $\mathrm{C}$ parameter - $\mathrm{A}$ smaller margin is accepted for larger $\mathrm{C}$ values if the decision function is better at correctly classifying all training points and also defines the high penalty for errors. A lower $\mathrm{C}$ actually encourages a greater margin, therefore a simpler decision function and for the accuracy of cost training [25-26].

b) The gamma parameter $(\gamma)$-This parameter determines the interaction between the error reduction and the smoothness of the function. The high gamma value demonstrates the correct fitting of the training data. The low value of gamma exemplifies the reduction of the model problem [27].

c) The sigma parameter, $\sigma 2$ - A higher value of sigma will show smooth and flexible in SVM decision function [27].

d) Kernel function $\mathrm{K}(\mathrm{x}, \mathrm{y})$ - SVM used non- linear mapping to map the training data points into a higher dimensional feature space. There are four types of kernel functions that can be used in SVM to construct mapping [21, 28-30]. In reference [19-21, 27-29], the RBF kernel is chosen as the best choice for the SVM kernel function because the RBF kernel has fewer numerical problems. The RBF kernel has low hyper- parameters than the polynomial kernel. Last but not least, RBF kernel maps samples into higher dimensional space non-linearly which not similar to the linear kernel. In references [31], the range of $\mathrm{C}$ chosen for training is $(1,100000)$ and ranges of gamma, $\gamma$ are $(0.005,20.0)$.

\subsection{Python Programming}

This paper uses Python as the programing language. Python's extensive machine learning library database and the support community are the main reasons of choice for this research. Python programing framework also has the ability to automate the process of training the models and interchanging the transformation methods. Figure 2 shows the flow chart of the proposed SVM algorithm in Python.

Pandas is highly recommended for large amounts of data to analyze. Pandas is an open-source Python library that provides high performance data analysis tools and easy to use data structures. The pandas work extremely well in Jupyter notebooks to share data, code, analysis results, visualizations, and narrative text. The pandas library has csv parsing capability as well to handle the data analysis. In scikit-learn, an estimator for classification is a Python object that can predict the classes to which the samples belong. An estimator is the class which implements support vector classification (SVC) [32].

Before the process of importing data into the Python framework, the data must be subjected to preprocessing. The preprocessing task was divided into formatting and cleaning up the sets of data. The format of the data file depended on the programing platform and the import library. Since Python was used to develop the algorithm, the data has to be in comma-separated values (CSV) extension file format [9]. Thus, in order to comply with the Python data reading standard, the original text file was converted into CSV by using Microsoft's Excel package. The converted CSV file was then subjected to the cleaning and screening process. During this process, each missing data was replaced with zero values so that the integrity of the data size was maintained.

The processed data were then imported to Python's framework by using Pandas library. The first part of the Python's data transformation framework was the data transformation module. Data transformation module is the library for all three-data transformation methods that were investigated in this paper. The way the transform module worked was by selecting one data transformation method for each run. During each run, two different model evaluations were tested which were split train and k-fold. Both of these validation methods have been widely used in classification based machine learning. The split train method is based on the principle theory in which data are randomly split to a certain percentage for testing and training the model. Meanwhile, k-fold allocates the data into a certain number of folds for training with the rest of the data for the training model. Unlike the split train, k-fold uses the whole data sets for training and testing. However, the method used does not over-fit the model, which makes k-fold the most sought-after method for classification accuracy validation.

For the split train model evaluation, the data were split for training and testing with a split percentage for testing and training. Meanwhile, $\mathrm{k}$ fold split the data into 18 folds which were used for both training and testing the transformed data. The model derived from the classifier-learning algorithm was then tested with the test data. The accuracy values were calculated and shown at the end of the process. 


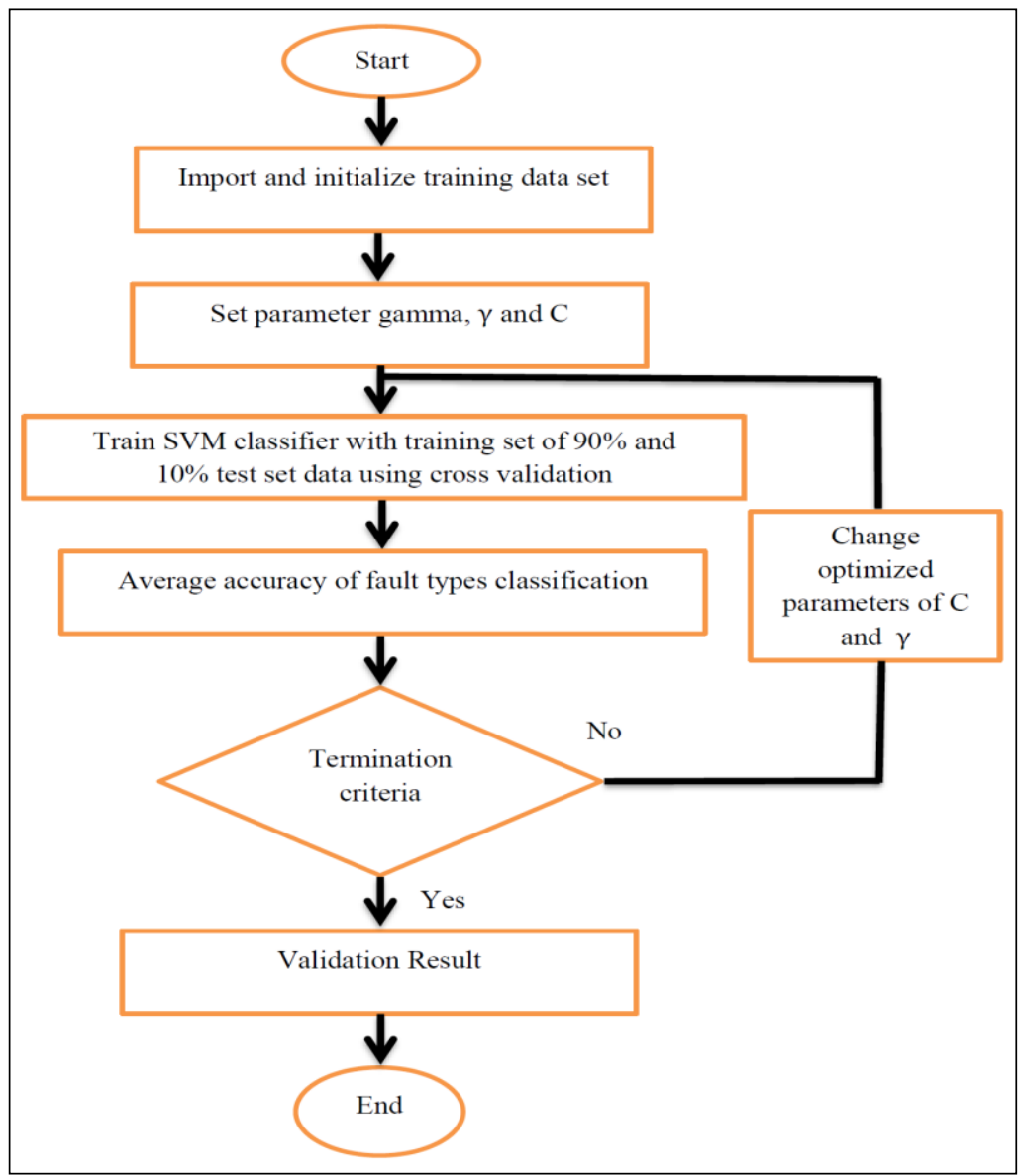

Figure 2. Flowchart of SVM Fault Classification in Python

\section{SIMULATION RESULTS}

The data used were obtained from the fault simulation on standard IEEE 34 and IEEE 123 bus test distribution system. IEEE 34 bus test system reflects an industry standard distribution test with a nominal voltage of $24.9 \mathrm{kV}$ located at Arizona. This system is characterized by long and lightly loaded, two in-line regulators, an in-line transformer for short $4.16 \mathrm{kV}$ section, unbalanced loading, and shunt capacitors [33-34]. IEEE 123 bus test system operates at a nominal voltage of $4.16 \mathrm{kV}$. It is characterized by overhead and underground lines, unbalanced loading with constant current, impedance, and power, four voltage regulators, shunt capacitor banks, and multiple switches [33-34].

The value of gamma, $\gamma$ and $C$ parameter values are set manually. In trial and error method, the SVM parameters, $\gamma$ and $\mathrm{C}$ were varied randomly in the training process. The value of $\mathrm{C}$ is kept constant when a high accuracy is achieved in this paper. The test size represented as the percentage separation of training data and testing data. In this paper, different test size are used for comparison result of accuracy, where test size of 0.1 is defined as $90 \%$ training data and $10 \%$ of testing data and respectively for test size of 0.2 . There are 48880 data for IEEE 34 bus and 1346310 data for IEEE 123 bus test system.

Table 1 show the fault type classification of IEEE 34 bus test distribution system. Based on Table 1, the classification accuracy is the highest when the test size ia 0.1 and the value of gamma is 0.01 with the highest classification accuracy of $99.99 \%$ for single line to ground fault type. On the other hand, the SVM training method takes longer computation time when gamma is 0.1 compare to 0.01 .

Table 2 show the fault type classification of IEEE 123 bus test distribution system. From Table 2, the classification accuracy is the highest when the test size is 0.1 and the value of gamma is 1 . The highest classification accuracy obtained is $99.08 \%$ for single line to ground fault type. Based on both tables, it is found that the accuracy result is higher when the number of training data is increased. However, there is no correlation observed for the value of gamma and C. Averagely, the classification accuracy for IEEE 34 bus system is higher compare to IEEE 123 bus system. This is because the number of bus is smaller hence higher classification accuracy is achieved easily. Nevertheless, the results show that the developed algorithm are able to classify the faults correctly on IEEE 123 bus test distribution system. 
Table 1. Fault Type Classification of IEEE 34 Bus System

\begin{tabular}{ccccc}
\hline Fault Types & \multicolumn{5}{c}{ Accuracy \% } \\
& \multicolumn{2}{c}{ Test Size $=0.1$} & Test Size $=0.2$ \\
& $\gamma=$ & $\gamma=$ & $\gamma=$ & $\gamma=$ \\
& 0.01 & 0.1 & 0.01 & 0.1 \\
\hline Single line to ground & 99.99 & 91.45 & 92.44 & 90.67 \\
D u le line to & 96.43 & 85.34 & 90.12 & 81.45 \\
ground & & & & \\
Line to line & 95.83 & 80.00 & 91.37 & 76.47 \\
Three phase & 80.77 & 76.44 & 80.11 & 70.12 \\
\hline
\end{tabular}

Table 2. Fault Type Classification of IEEE 123 Bus System

\begin{tabular}{cccccc}
\hline Fault Types & \multicolumn{5}{c}{ Accuracy \% } \\
& Test Size $=0.1$ & Test Size $=0.2$ \\
& $\gamma=0.1$ & $\gamma=1$ & $\gamma$ & .1 & $\gamma=1$ \\
\hline Single line to ground & 98.14 & 99.08 & 98.17 & 98.05 \\
Double line to ground & 72.78 & 91.89 & 83.92 & 80.12 \\
Line to line & 72.89 & 80.98 & 79.80 & 80.98 \\
Three phase & 80.65 & 81.21 & 72.49 & 72.55 \\
\hline
\end{tabular}

\section{CONCLUSION}

Fault type classification is important in power system protection and service restoration. Most research works have considered the fault diagnosis for overhead transmission systems but not many on distribution systems. The research done in distribution system mostly tested on small bus test distribution system. This paper has developed a machine learning algorithm known as SVM for fault classification in distribution system. SVM is chosen based on its capability of classifying different types of fault with high accuracy in a short period of time. The developed algorithm was tested in IEEE 34 and IEEE 123 bus test distribution system. Various test cases have been conducted in Python including variation of parameters gamma and test size using SVM technique to classify types of fault. Results show that the developed algorithm are able to classify types of fault with good accuracy at both IEEE 34 bus and IEEE 123 bus test distribution system.

In order to classify correctly with higher accuracy, a right combination of SVM parameter values are required. Try and error method is tedious, time consuming and does not guarantee the optimal combination of SVM parameters. The initial finding from this paper reveals that an automated or hybrid SVM algorithm to determine the optimal combination of SVM parameters are needed in order to obtain the highest fault classification accuracy.

\section{ACKNOWLEDGEMENTS}

The authors would like to thank Universiti Tenaga Nasional (UNITEN) through a BOLD research grant for the financial support to this research.

\section{REFERENCES}

[1] P. C. Paiva, H. M. Khodr, J. A. Domínguez-Navarro, J. M. Yusta, A. J. Urdaneta, "Integral Planning of PrimarySecondary Distribution Systems Using Mixed Integer Linear Programming," IEEE Trans. Power System, 2005, Vol. 20, pp. 1134-1143.

[2] H. A. I. S.S.Gururajapathy, H.Mokhlis, "Fault location and detection techniques in power distribution systems with distributed generation: A review," Renewable \& Sustainable Energy Review, 2017.

[3] Wester CG, "High impedance fault detection on distribution systems," 42nd IEEE Annual Conference Rural Electric Power Conference, 1998, pp. c5-1-5.

[4] Filomena AD, Resener M, Salim RH, et al., "Distribution systems fault analysis considering fault resistance estimation". International Journal of Electric Power Energy System, 2011, 33: 1326-1335.

[5] Lanz B, Sanchez E, "Is Fault Location Killing Our Cable Systems?" IEEE/PES Transmission and Distribution Conference and Exposition (T\&D), 2016, pp. 1-5.

[6] H. O. B. Karan Gandhi, "Smart Metering in electric power distribution system," International Conference of Control Automation Robot Embedded System, 2013.

[7] L. W. V. Devabhaktuni S. Depuru, "Smart meters for power grid: Challenges, issues, advantages and status," Renewable \& Sustainable Energy Review, Vol. 15(6), 2011, pp. 2736-2742.

[8] L. G. W. S. and J. R. S. M. M. Kezunovic R. A. F. Pereira, "Improved fault location on distribution feeders based on matching during-fault voltage sags," IEEE Transaction Power Delivery, 2009.

[9] D. K. J. Cordova N. Beneby T. Baldwin, "Fault locating in distribution networks with the aid of the advanced measurement infrastructure," Power System Conference Clemson University, 2004.

[10] C. S. Nicholas S. Coleman, "A Study of Power Distribution System Fault Classification with Machine Learning Techniques," North America Power Symposium, 2015.

[11] R. Salat, and S. Osowski, "Accurate fault location in the power transmission line using support vector machine approach," IEEE Transaction Power System, Vol. 19, no. 2, 2004.

[12] Papia Ray and B. K. Panigrahi, "Extreme learning machine based fault classification in a series compensated transmission line,” IEEE International Conference Power Electron Drives Energy System, 2012. 
[13] S. R. S. G. P. P. K. Dash, "Fault Classification and Section Identification of an Advanced Series-Compensated Transmission Line Using Support Vector Machine," IEEE Transaction Power Delivery, Vol. 22, No. 1, 2007.

[14] Ke Wang, Weirong Chen, and Qi Li, "Power System Fault Identification Method Based on Multi-wavelet Packet and Artificial Neural Network," Second International Conference Intelligent System Des. Eng. Appl., 2012.

[15] N Gana, N F Ab Aziz, Z Ali, H Hashim, Bahisham Yunus, "A Comprehensive Review of Fault Location Methods for Distribution Power System," Indonesian Journal of Electrical Engineering and Computer Science. 6. 185. 10.11591/ijeecs.v6.i1.pp185-192., 2017.

[16] V. N. Vapnik, "The Nature of Statistical Learning Theory,” New York Springer-Verlag, 2002.

[17] A. N, C. P, S. B. and B. Suechoey, "An Application of Discrete Wavelet Transform and Support Vector Machines Algorithm for Classification of Fault Types on Underground Cable," Third International Conference Innovation Bio-Inspired Computer Application, 2012.

[18] D. Thukaram; H.P. Khincha, "Artificial neural network and support vector Machine approach for locating faults in radial distribution systems," IEEE Transaction Power Delivery, Vol. 20, No. 2, 2005.

[19] D. Thukaram; H.P. Khincha, "Artificial neural network and support vector Machine approach for locating faults in radial distribution systems," IEEE Transaction Power Delivery, Vol. 20, No. 2, 2005.

[20] D. T. R. Agrawal, "Identification of fault location in power distribution system with distributed generation using support vector machines," 2013 IEEE PES Innovation Smart Grid Technology Conference, pp. 1-6, 2013.

[21] C. V. Ramón Pérez, "Fault Location in distribution systems with distributed generation using Support Vector Machines and smart meters," IEEE Ecuador Technical Chapters Meet., 2016.

[22] Deng X, Yuan R, Xiao Z, Li T, Wang KL. Fault location in loop distribution network using SVM technology. International Journal of Electrical Power \& Energy Systems. Feb 28 2015, 65:254-61.

[23] R. C. Dugan, "Reference Guide - The open distribution system simulator (OpenDSS)," EPRI.

[24] C. V. Ramón Pérez, "Fault Location in distribution systems with distributed generation using Support Vector Machines and smart meters," IEEE Ecuador Technical Chapters Meet, 2016.

[25] C. Q. H. M. Shunjie Han, "Parameter selection in SVM with RBF kernel function," World Autom. Congr., 2012.

[26] "RBF SVM parameters," scikit learn, 2017. [Online]. Available: https://scikitlearn.org/stable/auto_examples/svm/plot_rbf_parameters .html. [Accessed: 20-Dec-2018].

[27] C. L. B. Z. Weimin Zhang, "LSSVM Parameters Optimizing and Non-linear System Prediction Based on Cross Validation," Fifth International Conference Nat. Computing, 2009.

[28] Z.-F. H. ; X.-W. Y. Bo Liu, "Nesting support vector machine for multi-classification," International Conference Machine Learning Cybernatics, 2015.

[29] J. L. Chen Junli, "Classification Mechanism of Support Vector Machines," International Conference Signal Processing Proceeding, 2000.

[30] Z. M. Yasin, N. F. Ab Aziz, N. Salim, N. Wahab, N. Rahmat, "Optimal Economic Load Dispatch using Multiobjective Cuckoo Search Algorithm”. Indonesian Journal of Electrical Engineering and Computer Science. 12. 168-174. 10.11591/ijeecs.v12.i1.pp168-174., 2018.

[31] D. T. and H. P. K. B. Ravikumar, "Application of support vector machines for fault diagnosis in power transmission system," International Conference on Generation Transmission Distribution, Vol. 2, pp. 119-130, 2008.

[32] "Application for Python," Python Software Foundation, 2019. [Online]. Available: https://www.python.org/about/apps/. [Accessed: 19-Jan-2019].

[33] Kersting WH. Radial distribution test feeders. IEEE Transactions on Power Systems. August 1991, 6(3):975-85.

[34] Arvind Raj, Nur Fadilah Ab Aziz, Zuhaila Mat Yasin, Nur Ashida Salim, "Investigation of distributed generation units placement and sizing based on voltage stability condition indicator (VSCI)," International Journal of Power Electronics and Drive System (IJPEDS), Vol. 10, No. 3, Sep 2019, pp. 1317 1323. 